\title{
Variation in the Use of Advanced Imaging at the Time of Breast Cancer Diagnosis in a Statewide Registry
}

Running Head: Statewide Variation in Cancer Staging

N. Lynn Henry, MD. PhD ${ }^{1,2}$, Thomas M. Braun, $\mathrm{PhD}^{3}$, Tara M. Breslin, MD ${ }^{4}$, David H. Gorski, $\mathrm{MD}, \mathrm{PhD}^{5,6}$, Samuel M. Silver, $\mathrm{MD}, \mathrm{PhD}^{7}$, Jennifer J. Griggs, MD, $\mathrm{MPH}^{3,7}$

${ }^{1}$ Huntsman Cancer Institute

${ }^{2}$ University of Utah School of Medicine

${ }^{3}$ University of Michigan School of Public Health

${ }^{4}$ St. Joseph Mercy Health System, Ann Arbor

${ }^{5}$ Wayne State University School of Medicine

${ }^{6}$ Barbara Ann Karmanos Cancer Institute

${ }^{7}$ University of Michigan Medical School

\section{Corresponding Author:}

N. Lynn Henry

2000 Circle of Hope Drive

Huntsman Cancer Institute

Salt Lake City, UT 84112

801-587-9439

FAX 801-585-0124

\section{Manuscript details:}

Text Pages: 17

Figures: 2 (plus 3 supplemental figures)

Tables: 3 (plus 1 supplemental table)

Research Funding: The Michigan Breast Oncology Quality Initiative is a Blue Cross Blue Shield of Michigan (BCBSM)/Blue Care Network-supported Collaborative Quality Initiative. BCBSM provides funding to the participating hospitals for the data collection and analysis.

Prior Presentation: These findings were previously presented in part as a poster presentation at the 2016 ASCO Quality Care Symposium.

This is the author manuscript accepted for publication and has undergone full peer review but has not been through the copyediting, typesetting, pagination and proofreading process, which may lead to differences between this version and the Version record. Please cite this article as doi:10.1002/ cncr.30674. 
Conflicts of Interest: The authors have no relevant conflicts of interest to disclose.

\section{Author Contributions:}

Conceptualization: N Henry, S Silver, J Griggs

Software: T Braun

Formal analysis: $\mathrm{T}$ Braun

Investigation: N Henry, T Braun

Data curation: T Braun

Writing - original draft: N Henry

Writing - review and editing: N Henry, T Braun, S Silver, D Gorski, T Breslin, J Griggs

Visualization: N Henry, T Braun

Funding Acquisition: N Henry, S Silver

Condensed abstract: Use of advanced imaging at the time of diagnosis of stage 0-IIA breast cancer decreased between 2008 and 2015 in a large statewide collaborative, and remained stable for stage IIB disease. This decrease in testing over time resulted in a cost savings, especially for patients with stage I disease.

Keywords: breast cancer; CT scan; PET scan; bone scan; cost analysis; healthcare quality assessment; diagnostic imaging

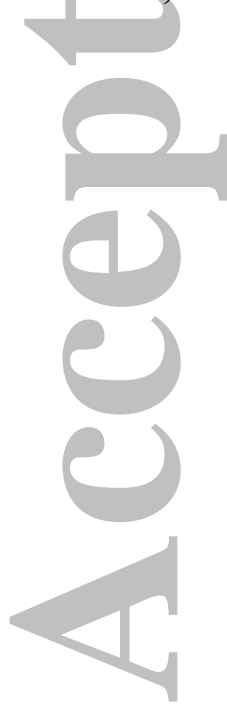




\section{Abstract}

Background: Although national guidelines do not recommend extent of disease imaging for patients with newly diagnosed early stage breast cancer because harms outweigh benefits, high rates of testing have been documented. The 2012 Choosing Wisely guidelines specifically addressed this issue. We examined the change over time in imaging use across a statewide collaborative, as well as the reasons for performing imaging and the impact on cost of care.

Methods: Clinicopathologic data and use of advanced imaging tests (CT, PET, and bone scan) were abstracted from the medical records of patients treated at 25 participating sites in the Michigan Breast Oncology Quality Initiative (MiBOQI). For patients diagnosed in 2014-15, reasons for testing were abstracted from the medical record.

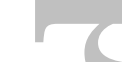

Results: Of the 34,078 patients diagnosed with stage 0-II breast cancer between 2008 and 2015 in MiBOQI, 6853 (20.1\%) underwent testing with at least one imaging modality in the 90 days following diagnosis. There was considerable variability in rates of testing across the 25 sites for all stages of disease. From 2008 to 2015 , testing decreased over time for patients with stage 0 IIA disease $($ all $\mathrm{p}<0.001)$ and remained stable for stage IIB disease $(\mathrm{p}=0.10)$. This decrease in testing over time resulted in a cost savings, especially for patients with stage I disease.

Conclusions: Use of advanced imaging at the time of diagnosis decreased over time in a large statewide collaborative. Additional interventions are warranted to further reduce rates of unnecessary imaging in order to improve quality of care for patients with breast cancer. 


\section{Introduction}

In 2012 the American Society of Clinical Oncology, in conjunction with the American Board of Internal Medicine Foundation, released the first of a series of guidelines called Choosing Wisely to educate patients and providers about unnecessary procedures. ${ }^{1}$ One of the so called "Top 5"

for oncology recommended against the use of advanced imaging with positron emission tomography (PET), computed tomography $(\mathrm{CT})$, and radionuclide bone scans in asymptomatic patients with newly diagnosed early stage (stage I and II) breast cancer for the purpose of detecting metastatic disease.

The American Society of Clinical Oncology and National Comprehensive Cancer Network (NCCN) also both recommend against use of advanced imaging for assessment of patients with stage I and II breast cancer if they have no signs or symptoms concerning for metastatic disease. ${ }^{2,3}$ The rationale behind these recommendations is the low likelihood of the presence of metastatic disease in asymptomatic patients with newly diagnosed stage I and II breast cancer. ${ }^{4-7}$ In contrast, the NCCN guidelines recommend considering assessment with advanced imaging for patients with clinical stage III disease given the higher prevalence of distant metastatic disease in these patients.

In addition to a low potential for benefit from imaging in asymptomatic patients with stage I and II breast cancer, there are also considerable harms. Advanced imaging generally requires radiation, and cumulative radiation exposure can increase the risk of second malignancies. ${ }^{8}$ In addition, there are often false positive or indeterminate findings on extent of disease evaluation, which can cause anxiety, and can lead to the need for invasive biopsies as well as subsequent 
scans for further evaluation. Other potential harms from imaging are delays in care and increased healthcare costs. It is therefore essential to minimize the inappropriate use of advanced imaging in this patient population.

In order to evaluate the variation in use of CT chest, abdomen, and pelvis, PET, and bone scan imaging across a single state over time, the Michigan Breast Oncology Quality Initiative (MiBOQI) collected data regarding use of advanced imaging in a prospective registry of 25 participating hospitals. In addition, clinical and non-clinical factors associated with test usage were examined. We hypothesized that use of advanced imaging over time has decreased, especially since the publication of the Choosing Wisely initiative.

\section{Methods}

Patient characteristics

MiBOQI is a Blue Cross Blue Shield of Michigan/Blue Care Network-supported Collaborative Quality Initiative comprising 25 hospital systems that abstract comprehensive demographic, clinical, and pathologic data on patients with newly diagnosed breast cancer. The members of the collaborative conduct quality initiative projects, with the goal of improving the care of patients with breast cancer across the state of Michigan. Data from patients with stage 0 -II breast cancer who were diagnosed between 2008 and 2015 and treated at one of the 25 participating MiBOQI hospitals were abstracted from the medical record and included in the registry. If a patient was treated with primary systemic therapy, the staging was based on clinical stage at presentation. If a patient was treated with primary surgery, the staging was based on pathologic stage.

All patients who present to a participating site within 180 days of diagnosis of a new breast cancer and who undergo surgery and/or systemic therapy at that institution are included in the 
registry, except for patients with male gender, age under 18, and a history of a non-breast invasive malignancy diagnosed within 90 days of the breast cancer. If a patient receives treatment at two MiBOQI institutions, she is included in the cohort where she received her first cancer-directed treatment.

Diagnosis of breast cancer was based on the date of the initial biopsy that demonstrated cancer.

Data elements that were abstracted included demographic characteristics, pathologic findings, and treatments administered. In addition, dates of all CT, PET, and bone scans performed within 90 days following diagnosis of breast cancer were abstracted, except for scans that were performed for reasons deemed by the data abstractor to be unrelated to the diagnosis and evaluation of breast cancer. Scans performed prior to the date of diagnosis were excluded because the intent was to capture only those scans done as a result of the breast cancer diagnosis. For the cohort of patients diagnosed in 2014-2015, reasons for testing were abstracted from the medical record using a pre-defined list of reasons. These reasons were divided into reasons considered concordant or non-concordant with guidelines by the authors of the manuscript prior to data analysis; the data abstractors were not aware of the classification of each reason.

\section{Statistical analysis}

All data were analyzed using the statistical package R, version 3.2.3. Overall stage was based on a combination of clinical and pathologic stage. For those patients who underwent primary surgical resection, pathologic staging (including $\mathrm{T}$ and $\mathrm{N}$ stage) was used. In contrast, for those patients who received treatment with primary systemic therapy, clinical staging was used.

All patient characteristics were summarized as proportions, and the statistical significance of how scan utilization varied with patient characteristics was assessed using univariate and 
multivariate logistic regression. Cost savings were computed using scan costs for 2011 listed by the Centers for Medicare \& Medicaid Services (CMS) (average cost of CT \$400, PET \$1075, and bone scan \$290) applied to scan totals per 1000 patients observed in our data in 2010-2011 and 2015. Statistical significance was defined as a p-value less than 0.05 .

\section{Results}

\section{Use of advanced imaging studies}

Of the 34,078 patients diagnosed with stage 0-II breast cancer between 2008-2015 and included in the registry, 6853 (20.1\%) patients underwent imaging with CT, PET, and/or bone scan for any reason within 90 days following diagnosis of breast cancer (Supplemental Figure 1). The percentage of those who underwent testing with at least one imaging modality increased with increasing stage (Figure 1A). Similarly, the number of scans performed per patient increased with increasing stage (Supplemental Figure 2). In particular, almost 25\% of patients with stage IIB disease underwent at least 3 advanced imaging tests, compared to approximately $12 \%$ for patients with stage IIA disease and less than $5 \%$ for stages 0 and I. Use of each type of imaging modality varied. CT scan was used most commonly, with 4953 (14.5\%) patients undergoing at least one scan within 90 days following diagnosis of breast cancer. At least one bone scan was used for the assessment of 3268 (9.6\%) patients. PET scans were used least frequently, with $1602(4.7 \%)$ patients undergoing at least one scan in the 90 days following diagnosis.

The number of patients who underwent imaging with at least one diagnostic scan decreased over time from 2008 to 2015 for stage 0 -IIA breast cancer (all $\mathrm{p}<0.001$ ), and remained stable for stage IIB disease $(\mathrm{p}=0.10)$ (Figure 1A, Supplemental Table 1). When specific imaging modalities 
were examined, the number of patients who underwent imaging with at least one CT scan or at least one bone scan decreased for stage 0-IIA breast cancer $(\mathrm{p}<0.001)$ (Figures $1 \mathrm{~B}$ and $1 \mathrm{C})$. For PET scans, the number of patients who underwent imaging between 2008 and 2015 decreased for stages I and IIA breast cancer $(p=0.001)$ but there was no apparent significant decrease for patients with stages 0 and IIB disease (Figure 1D).

\section{Variability across MiBOQI sites}

Across the 25 participating sites, there was considerable variability in the use of imaging tests (Figure 2). When examining the number of patients with stage 0 disease who underwent any advanced imaging scan during the 90 days following diagnosis, rates of testing varied from $2.5 \%$ to $43.7 \%$, with a median of $5.5 \%$. For stages I, IIA, and IIB, the medians were $13.0 \%(7.6$ $55.9 \%), 30.4 \%(15.2-66.5 \%)$, and $53.1 \%(27.5-84.9 \%)$, respectively.

The majority of scans performed were CT scans. When examining the number of patients with stage 0 disease who underwent any CT scan, rates of testing across the sites varied from $1.3 \%$ to $43.7 \%$, with a median of $4.6 \%$ (Supplemental Figure 3A). For stage I, IIA, and IIB, the medians were $9.5 \%(4.6-53.9 \%), 18.3 \%(8.3-61.5 \%)$, and $32.6 \%(10.6-69.9 \%)$, respectively (Supplemental Figures 3B-D).

Fewer patients underwent testing with bone scans or PET scans. When examining the number of patients with stage 0 disease who underwent a bone scan, rates of testing across the sites varied from $0 \%$ to $19.7 \%$, with a median of $0.7 \%$ (Supplemental Figure $3 \mathrm{~A}$ ). For stage I, IIA, and IIB, the medians were $3.8 \%(0-47.0 \%), 10.7 \%(2.7-56.0 \%)$, and $27.8 \%(0-64.0 \%)$, respectively (Supplemental Figures 3B-D). For PET scans, the median for stage 0 was $0.4 \%$ (range $0-2.5 \%$ ), 
for stage I was $1.8 \%(0.7-8.2 \%)$, for stage IIA was $8.3 \%(1.0-37.3 \%)$, and for stage IIB was $16.2 \%(2.8-64.3 \%)$ (Supplemental Figure 3). The participating sites with the highest rates of testing with CT differed from those with the highest rates of testing with PET.

\section{Associations between patient characteristics and advanced imaging}

Univariate and multivariate analyses were performed to assess associations between patient and pathologic characteristics and test ordering (Table 1). On univariate analysis, younger age, black race, lower socioeconomic status, higher comorbidity score, and higher clinical stage were associated with higher likelihood of advanced imaging. Pathologic characteristics associated with advanced imaging on univariate analysis included lack of ER expression, HER2 overexpression or amplification, higher tumor grade, larger tumor size, and greater number of involved lymph nodes. On multivariate analyses, all factors were statistically significant except for Charlson comorbidity index, mostly due to its correlation with several other patient factors.

\section{Reasons for testing}

The medical records of patients from 2014-2015 were examined to determine reasons for testing (Table 2). Of the 1687 patients who had advanced imaging performed, 55.1\% had imaging performed for reasons considered to be concordant with guidelines; the remaining $44.9 \%$ had tests performed for reasons considered non-concordant or not documented in the medical record.

For patients with stage 0 disease, $4.4 \%$ had at least one advanced imaging test performed, of which $81 \%$ were considered to be concordant. Of those patients who underwent imaging, more 
than half did so to evaluate patient-reported symptoms, for a non-breast cancer-related condition, or for follow-up of an abnormal test. Twenty percent of tests were ordered by outside providers for unknown reasons, and an additional 19\% didn't have a clearly documented reason.

For patients with stage I disease, $10.3 \%$ had at least one advanced imaging test performed, of which $69 \%$ were considered to be concordant. Of those who were tested, $23 \%$ of patients underwent imaging to evaluate patient-reported symptoms, $21 \%$ for follow-up of an abnormal test, and $4 \%$ because of clinicopathologic features that do not clearly indicate the need for imaging, such as abnormal-appearing lymph nodes, large tumor size based on imaging, young age, and triple negative disease. Nine percent of tests were ordered by outside providers for unknown reasons, and an additional $26 \%$ didn't have a clear reason documented in the medical record.

For patients with stage IIA and IIB disease, $33.8 \%$ had at least one advanced imaging test performed. For those with stage IIA disease, 52\% were considered to be concordant, as opposed to $47 \%$ of with stage IIB disease. Of those patients who were tested, fewer underwent imaging because of patient-reported symptoms or follow-up of an abnormal test compared to patients with stage I disease. About one-fifth of patients had testing prior to initiation of neoadjuvant chemotherapy. Compared to stage I disease, a higher percentage of patients underwent evaluation with advanced imaging because of node positive disease that didn't meet the criteria for clinical stage III disease (14-26\%). About one-quarter of tests were performed for reasons not clearly documented in the medical record.

Cost impact of decreased testing over time 
The cost of advanced imaging tests performed in patients diagnosed in 2010-11, prior to the publication of the Choosing Wisely recommendations, were compared to the cost of those performed in patients diagnosed in 2015. As shown in Table 3, using the average cost for the scans based on the CMS fee schedule from 2011 there was a 33\% decrease over time in the total cost of scans for patients with stage 0 -II breast cancer. When divided by stage, there was a trend towards a greater savings for those with stage I disease (36\%) compared to stage 0 or II (16\% and $23 \%$, respectively). Similar trends were identified when each individual imaging modality was examined.

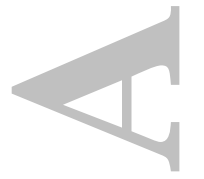

\section{Discussion}

In this large statewide registry, use of advanced imaging within 90 days of diagnosis of stage 0IIA breast cancer decreased between 2008 and 2014. As expected, higher rates of imaging were seen in patients at higher risk of disease recurrence. There was considerable variability in use of imaging across the 25 participating hospital systems, which varied by both disease stage and imaging modality. Symptoms, abnormalities identified on other testing, or other disease conditions were identified as the reasons for testing for about half of patients with stage 0 or I disease. In contrast, factors associated with more aggressive disease were more commonly cited as reasons for testing in those with stage II disease. These findings support and extend those previously reported in the literature. ${ }^{9-13}$

The Choosing Wisely recommendations were developed to reduce the use of advanced imaging in asymptomatic patients with newly diagnosed stage 0 -II breast cancer because of the minimal benefits and potential harms of testing. The likelihood of having metastatic disease in this setting 
has been demonstrated to be less than $2 \%$ in multiple studies. ${ }^{14,15}$ Harms from performing unnecessary imaging can include the cost of testing to patients and society, radiation exposure from the imaging tests, and anxiety related to testing.

One additional harm that can arise is the potential need to expose patients to additional radiation and/or invasive procedures in order to evaluate abnormal findings. ${ }^{8}$ However, in the MiBOQI registry we did not collect downstream effects of imaging, including frequency of abnormal scan results or use of additional imaging or biopsies. Both of these can increase risk to patients as well as increase the cost of care. As recently reported in a study of patients with stage II and III breast cancer, more than $80 \%$ of patients had an abnormality noted on advanced imaging, and $43 \%$ underwent additional evaluation. ${ }^{14}$

Use of testing for patients with stage IIB disease remained high throughout the study period. Although asymptomatic patients with stage IIB disease were included in the Choosing Wisely recommendations, it is unclear whether these patients should be excluded from routine extent of disease evaluation. These patients are at increased risk of having metastatic disease compared to those with lower stage breast cancer. In addition, many patients with stage IIB disease undergo treatment with primary systemic therapy, and therefore their actual extent of disease is unknown at the time of imaging, assuming imaging is performed prior to treatment initiation. Routine use of imaging in this population may therefore need to be subjected to further study.

The reduction in testing within the first 90 days after diagnosis that occurred between 2010-11 and 2015 resulted in substantial cost savings overall based on the Medicare fee schedule, especially in patients with stage I breast cancer. Because of limitations in the registry we were unable to account for downstream imaging or biopsies that resulted from initial extent of disease 
evaluation. Therefore, because of this limitation and the use of Medicare reimbursement rates rather than private payer rates, the cost savings is likely greater than was identified in this analysis.

Rates of testing decreased at the 25 participating sites across Michigan during the time period that the Choosing Wisely recommendations were published. However, it is unknown what led to this reduction. One possibility is the Choosing Wisely campaign itself and the associated media coverage, although rates of testing in Michigan appeared to start decreasing prior to the publication. In addition, others have demonstrated minimal difference in use of testing before and after the publication. ${ }^{11}$ Furthermore, numerous studies have demonstrated a significant lag in the uptake of new guidelines or findings by physicians following their initial publication or presentation. ${ }^{16}$ Therefore it is possible that practice patterns changed in Michigan because of increased awareness of MiBOQI participating physicians related to regular discussions of the topic at MiBOQI's Tri-Annual meetings, and subsequent dissemination of the information to colleagues at tumor board conferences. A formal collaborative quality initiative was launched in May 2013 although the topic was discussed at meetings during the year prior to that time. A final possibility is the requirement for prior authorization of advanced imaging tests by insurance companies, which may have resulted in fewer imaging tests being performed although may not have altered the actual ordering of scans by providers.

In 2014-15,20-30\% of imaging tests ordered for patients with stage 0 -II disease did not have a clearly documented reason in the medical record. It is possible that almost all of the testing performed in these more recent years was guideline-concordant, but it is difficult to tell because of incomplete documentation. Using strategies such as multidisciplinary tumor conference case reviews and decision making tools incorporated into the electronic health record could 
potentially help reduce unnecessary test ordering; the latter has been used for prevention of venous thromboembolic disease and reducing overuse of antibiotics. ${ }^{17-19}$

The variability of testing across participating hospital systems was also notable, and similar to what has previously been reported in the literature. $9,13,20$ Those sites with the highest percentage of non-concordant testing of patients with stage II disease treated fewer patients compared to those with lower rates of testing, although numerous other sites that treated smaller numbers of patients also had low rates of testing. The reasons for the variation are unknown but could be related to differences in local practice patterns, patient mix across practice sites, lack of a multidisciplinary tumor board, concerns about litigation, or financial pressures.

Overall, these findings represent changes in frequency of imaging at the time of breast cancer diagnosis that have occurred across a single state during the time frame that spanned publication of the Choosing Wisely guidelines. Our results are based on a large registry of patients treated in a variety of practice settings. Although we used a pre-defined list when assessing reasons for testing, the data are limited since they were collected retrospectively by data abstractors with physician involvement as needed, and not prospectively at the time of ordering.

In summary, within MiBOQI the rate of imaging within 90 days of diagnosis of stage 0-IIA breast cancer decreased significantly between 2008 and 2015, although imaging in stage IIB disease remained relatively stable. This decrease likely reduced exposure to multiple harms, including radiation exposure, unnecessary invasive procedures, and financial toxicity. Although great strides have been made to date, additional interventions are required to reduce the rates of unnecessary testing even further, thereby improving the quality of care for patients with breast cancer. 


\section{Acknowledgements}

We thank the Clinical Data Abstractors and Clinical Champions at the 25 MiBOQI participating sites for their assistance with collecting the data for this analysis. We also thank Tiffani Stewart for working with the Clinical Data Abstractors to obtain the data on reasons for testing.
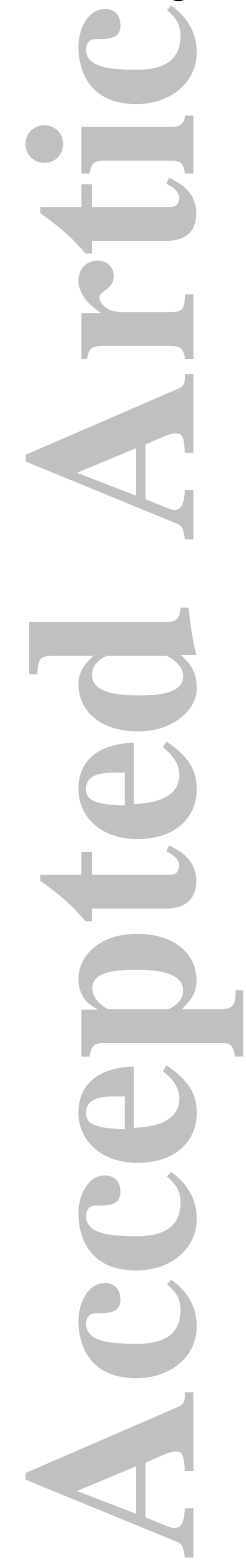


\section{References}

1. Schnipper LE, Smith TJ, Raghavan D, et al. American Society of Clinical Oncology identifies five key opportunities to improve care and reduce costs: the top five list for oncology. J Clin Oncol. 2012;30: 1715-1724.

2. Khatcheressian JL, Hurley P, Bantug E, et al. Breast cancer follow-up and management after primary treatment: American Society of Clinical Oncology Clinical Practice Guideline update. J Clin Oncol. 2013;31: 961-965.

3. Gradishar WJ, Anderson BO, Balassanian R, et al. Breast cancer, version 1.2016. J Natl Compr Canc Netw. 2015;13: 1475-1485.

4. Puglisi F, Follador A, Minisini AM, et al. Baseline staging tests after a new diagnosis of breast cancer: further evidence of their limited indications. Ann Oncol. 2005;16: 263-266.

5. Kasem AR, Desai A, Daniell S, Sinha P. Bone scan and liver ultrasound scan in the preoperative staging for primary breast cancer. Breast J. 2006;12: 544-548.

6. Gerber B, Seitz E, Muller H, et al. Perioperative screening for metastatic disease is not indicated in patients with primary breast cancer and no clinical signs of tumor spread. Breast Cancer Res Treat. 2003;82: 29-37.

7. Kim H, Han W, Moon HG, et al. The value of preoperative staging chest computed tomography to detect asymptomatic lung and liver metastasis in patients with primary breast carcinoma. Breast Cancer Res Treat. 2011;126: 637-641.

8. Brenner DJ, Hall EJ. Computed tomography--an increasing source of radiation exposure. N Engl J Med. 2007;357: 2277-2284. 
9. Hahn EE, Tang T, Lee JS, et al. Use of imaging for staging of early-stage breast cancer in two integrated health care systems: adherence with a choosing wisely recommendation. J Oncol Pract. 2015;11: e320-328.

10. Kamal A, Zhang T, Power S, Marcom PK. Is advanced imaging in early-stage breast cancer ever warranted? Reconciling clinical judgment with common quality measures. J Natl Compr Canc Netw. 2016;14: 993-998.

11. Simos D, Hutton B, Clemons M. Are physicians choosing wisely when imaging for distant metastases in women with operable breast cancer? J Oncol Pract. 2015;11: 62-68.

12. Ramsey SD, Fedorenko C, Chauhan R, et al. Baseline Estimates of Adherence to American Society of Clinical Oncology/American Board of Internal Medicine Choosing Wisely Initiative Among Patients With Cancer Enrolled With a Large Regional Commercial Health Insurer. J

Oncol Pract. 2015;11: 338-343.

13. Rocque GB, Williams CP, Jackson BE, et al. Choosing Wisely: Opportunities for Improving Value in Cancer Care Delivery? J Oncol Pract. 2017;13: e11-e21.

14. Brothers JM, Kidwell KM, Brown RK, Henry NL. Incidental radiologic findings at breast cancer diagnosis and likelihood of disease recurrence. Breast Cancer Res Treat. 2016;155: 395403.

15. Brennan ME, Houssami N. Evaluation of the evidence on staging imaging for detection of asymptomatic distant metastases in newly diagnosed breast cancer. Breast. 2012;21: 112-123. 16. Grol R, Grimshaw J. From best evidence to best practice: effective implementation of change in patients' care. Lancet. 2003;362: 1225-1230.

17. Raja AS, Gupta A, Ip IK, Mills AM, Khorasani R. The use of decision support to measure documented adherence to a national imaging quality measure. Acad Radiol. 2014;21: 378-383. 
18. Goldzweig CL, Orshansky G, Paige NM, et al. Electronic Health Record-Based Interventions for Reducing Inappropriate Imaging in the Clinical Setting: A Systematic Review of the Evidence. Washington (DC): Department of Veterans Affairs (US), 2015.

19. Litvin CB, Ornstein SM, Wessell AM, Nemeth LS, Nietert PJ. Use of an electronic health record clinical decision support tool to improve antibiotic prescribing for acute respiratory infections: the ABX-TRIP study. J Gen Intern Med. 2013;28: 810-816.

20. Makarov DV, Soulos PR, Gold HT, et al. Regional-Level Correlations in Inappropriate Imaging Rates for Prostate and Breast Cancers: Potential Implications for the Choosing Wisely Campaign. JAMA Oncol. 2015;1: 185-194.

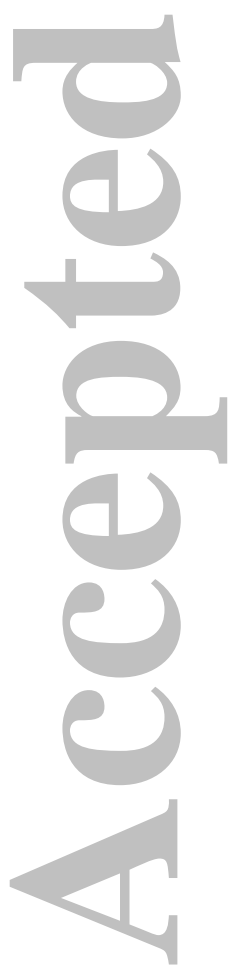




\section{Figure Legends}

Figure 1. Percentage of patients who had at least one scan performed, by year. Each line represents a different disease stage (blue: stage 0, red: stage I, green: stage IIA, purple: stage IIB). A) At least one scan of any imaging modality. B) At least one CT scan. C) At least one bone scan. D) At least one PET scan.

Figure 2. Percentage of patients who had at least one scan performed, by participating site. Sites are listed on the $\mathrm{x}$ axis from 1-25. Bars represent different disease stages (blue: stage 0, red: stage I, green: stage IIA, purple: stage IIB).

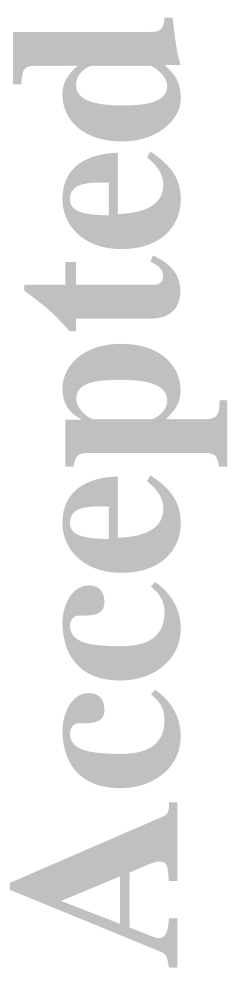


Table 1. Patient and pathologic characteristics associated with use of advanced imaging.

\begin{tabular}{|c|c|c|c|c|c|}
\hline & & $\begin{array}{c}\text { Scan } \\
\mathrm{N}=6853\end{array}$ & $\begin{array}{c}\text { No Scan } \\
\mathrm{N}=27,225\end{array}$ & $\begin{array}{l}\text { Univariate } \\
\mathrm{P} \text { value }\end{array}$ & $\begin{array}{c}\text { Multivariate } \\
\text { P value }\end{array}$ \\
\hline \multirow[t]{3}{*}{ Age } & $<50$ & $1740(25 \%)$ & $5188(75 \%)$ & $<0.001$ & 0.020 \\
\hline & $50-69$ & $3503(19 \%)$ & $14660(81 \%)$ & & \\
\hline & $70+$ & $1610(18 \%)$ & $7377(82 \%)$ & & \\
\hline \multirow[t]{4}{*}{ Race/ Ethnicity } & White & $5190(19 \%)$ & $22243(81 \%)$ & $<0.001$ & $<0.001$ \\
\hline & Black & $1163(27 \%)$ & $3190(73 \%)$ & & \\
\hline & Other & $108(26 \%)$ & $308(74 \%)$ & & \\
\hline & Hispanic & $392(21 \%)$ & $1484(79 \%)$ & & \\
\hline \multirow{4}{*}{$\begin{array}{l}\text { Socioeconomic } \\
\text { status (tertiles) }\end{array}$} & High & $1866(17 \%)$ & $9296(83 \%)$ & $<0.001$ & $<0.001$ \\
\hline & Medium & $2326(21 \%)$ & $8818(79 \%)$ & & \\
\hline & Low & $2563(23 \%)$ & $8696(77 \%)$ & & \\
\hline & Missing & $98(19 \%)$ & $415(81 \%)$ & & \\
\hline \multirow[t]{6}{*}{ Clinical stage } & 0 & $476(7 \%)$ & $6528(93 \%)$ & $<0.001$ & $<0.001$ \\
\hline & I & $2734(17 \%)$ & $13211(83 \%)$ & & \\
\hline & II & $2906(40 \%)$ & $4365(60 \%)$ & & \\
\hline & III & $99(77 \%)$ & $29(23 \%)$ & & \\
\hline & IV & $7(88 \%)$ & $1(12 \%)$ & & \\
\hline & Missing & $631(17 \%)$ & $3091(83 \%)$ & & \\
\hline \multirow[t]{6}{*}{ Path tumor stage } & pT1 & $3085(17 \%)$ & $14640(83 \%)$ & $<0.001$ & $<0.001$ \\
\hline & pT2 & $1810(30 \%)$ & $4169(70 \%)$ & & \\
\hline & pT3 & $116(46 \%)$ & $135(54 \%)$ & & \\
\hline & pT4 & $3(50 \%)$ & $3(50 \%)$ & & \\
\hline & Other & $543(7 \%)$ & $7536(93 \%)$ & & \\
\hline & Missing & $1296(64 \%)$ & $742(36 \%)$ & & \\
\hline \multirow{4}{*}{ Nodal involvement } & pN0 & $3473(15 \%)$ & $19416(85 \%)$ & $<0.001$ & $<0.001$ \\
\hline & $\mathrm{pN} 1 \mathrm{mi}$ & $245(25 \%)$ & $747(75 \%)$ & & \\
\hline & $\mathrm{pN} 1$ & $1425(39 \%)$ & $2191(61 \%)$ & & \\
\hline & $\mathrm{pN} 2$ & $5(100 \%)$ & $0(0 \%)$ & & \\
\hline
\end{tabular}




\begin{tabular}{|llcclc|}
\hline & Missing & $1705(26 \%)$ & $4871(74 \%)$ & & \\
\hline & 1 & $1213(17 \%)$ & $6111(83 \%)$ & $<0.001$ & $<0.001$ \\
Grade & 2 & $2610(23 \%)$ & $8927(77 \%)$ & & \\
& 3 & $2412(34 \%)$ & $4735(66 \%)$ & & \\
& Missing & $618(8 \%)$ & $7452(92 \%)$ & & \\
\hline ER positive & & $5156(75 \%)$ & $23122(85 \%)$ & $<0.001$ & $<0.001$ \\
\hline HER2 positive & & $1148(18 \%)$ & $2220(11 \%)$ & $<0.001$ & $<0.001$ \\
\hline & 0 & $5309(20 \%)$ & $21661(80 \%)$ & 0.001 & $>0.99$ \\
Charlson & 1 & $745(21 \%)$ & $2733(79 \%)$ & & \\
Comorbidity Index & 2 & $508(21 \%)$ & $1862(79 \%)$ & & \\
& $\geq 3$ & $291(23 \%)$ & $969(77 \%)$ & & \\
\hline
\end{tabular}


Table 2. Reasons for testing, according to medical record documentation, 2014-15. Percentages given are percent of the total number of patients with that stage of disease who had imaging performed.

\begin{tabular}{|c|c|c|c|c|}
\hline Reason & $\begin{array}{c}\text { Stage } 0 \\
\mathrm{~N}=54\end{array}$ & $\begin{array}{l}\text { Stage I } \\
\mathrm{N}=423\end{array}$ & $\begin{array}{l}\text { Stage IIA } \\
\quad \mathrm{N}=569\end{array}$ & $\begin{array}{c}\text { Stage IIB } \\
\mathrm{N}=641\end{array}$ \\
\hline Concordant & $44(81 \%)$ & $291(69 \%)$ & $295(52 \%)$ & $299(47 \%)$ \\
\hline Patient-reported symptom & $12(22 \%)$ & $96(23 \%)$ & $55(10 \%)$ & $29(5 \%)$ \\
\hline $\begin{array}{l}\text { Evaluation of abnormal imaging } \\
\text { study }\end{array}$ & $5(9 \%)$ & $55(13 \%)$ & $32(6 \%)$ & $29(5 \%)$ \\
\hline Evaluation of abnormal lab test & $1(2 \%)$ & $33(8 \%)$ & $18(3 \%)$ & $14(2 \%)$ \\
\hline Clinical stage III or IV & 0 & 0 & $13(2 \%)$ & $29(5 \%)$ \\
\hline $\begin{array}{l}\text { Pathologic stage III (but clinically } \\
\text { lower stage at diagnosis) }\end{array}$ & 0 & $6(1 \%)$ & 0 & $1(0.2 \%)$ \\
\hline Neoadjuvant chemotherapy & 0 & $13(3 \%)$ & $104(18 \%)$ & $149(23 \%)$ \\
\hline In anticipation of reconstruction & $4(7 \%)$ & $14(3 \%)$ & $8(1 \%)$ & $2(0.3 \%)$ \\
\hline Testing required by clinical trial & 0 & 0 & $1(0.1 \%)$ & $1(0.2 \%)$ \\
\hline Outside provider & $11(20 \%)$ & $40(9 \%)$ & $46(8 \%)$ & $36(6 \%)$ \\
\hline Unrelated condition & $9(17 \%)$ & $27(6 \%)$ & $15(3 \%)$ & $6(0.9 \%)$ \\
\hline Lung cancer screening & $2(4 \%)$ & $3(0.7 \%)$ & $3(0.5 \%)$ & $3(0.5 \%)$ \\
\hline $\begin{array}{l}\text { Uncertain recurrence vs new } \\
\text { primary }\end{array}$ & 0 & $4(0.9 \%)$ & 0 & 0 \\
\hline \multicolumn{5}{|l|}{4} \\
\hline Non-concordant & $10(19 \%)$ & $132(31 \%)$ & $274(48 \%)$ & $342(53 \%)$ \\
\hline Recommended by tumor board & 0 & $2(0.5 \%)$ & $6(1 \%)$ & $1(0.2 \%)$ \\
\hline Not documented & $10(19 \%)$ & $110(26 \%)$ & $169(30 \%)$ & $134(21 \%)$ \\
\hline \multicolumn{5}{|l|}{$\begin{array}{l}\text { Factors that do not meet guideline } \\
\text { criteria for imaging }\end{array}$} \\
\hline $\begin{array}{ll}\text { - } & \text { Node positive } \\
\text { disease/abnormal } \\
\text { appearing lymph nodes }\end{array}$ & 0 & $8(2 \%)$ & $81(14 \%)$ & $166(26 \%)$ \\
\hline - ER/PR/HER2 negative & 0 & $2(0.5 \%)$ & $6(1 \%)$ & $6(0.9 \%)$ \\
\hline - $\quad$ Large tumor size & 0 & $3(0.7 \%)$ & $6(1 \%)$ & $21(4 \%)$ \\
\hline - Bilateral disease & 0 & $4(0.9 \%)$ & $6(1 \%)$ & 4 \\
\hline - Young age & 0 & $1(0.2 \%)$ & 0 & $5(0.8 \%)$ \\
\hline Patient requested & 0 & $2(0.5 \%)$ & 0 & $5(0.8 \%)$ \\
\hline
\end{tabular}


Table 3. Cost savings analysis comparing the cost of advanced imaging scans performed within 90 days of diagnosis per 1000 patients in 2010-11 and 2015, using estimated costs from the 2011 Medicare fee schedule.

\begin{tabular}{|c|c|c|c|c|}
\hline $\begin{array}{l}\text { Imaging } \\
\text { modality }\end{array}$ & Stage & $\begin{array}{c}\text { Total cost } 2010- \\
11 \text { per } 1000 \\
\text { patients }\end{array}$ & $\begin{array}{c}\text { Total cost } 2015 \\
\text { per } 1000 \text { patients }\end{array}$ & $\begin{array}{c}\text { Percentage } \\
\text { reduction in cost }\end{array}$ \\
\hline \multirow{4}{*}{ All } & All & $\$ 202,400$ & $\$ 135,754$ & $33 \%$ \\
\hline & 0 & $\$ 40,133$ & $\$ 33,536$ & $16 \%$ \\
\hline & I & $\$ 141,543$ & $\$ 90,643$ & $36 \%$ \\
\hline & II & $\$ 433,007$ & $\$ 333,249$ & $23 \%$ \\
\hline \multirow{4}{*}{ CT scan } & All & $\$ 113,494$ & $\$ 75,245$ & $34 \%$ \\
\hline & 0 & $\$ 33,500$ & $\$ 25,051$ & $25 \%$ \\
\hline & I & $\$ 83,112$ & $\$ 47,741$ & $43 \%$ \\
\hline & II & $\$ 227,826$ & $\$ 152,981$ & $33 \%$ \\
\hline \multirow{4}{*}{$\begin{array}{l}\text { Bone } \\
\text { scan }\end{array}$} & $\overline{\text { All }}$ & $\$ 33,485$ & $\$ 17,473$ & $48 \%$ \\
\hline & 0 & $\$ 3,056$ & $\$ 1,465$ & $52 \%$ \\
\hline & I & $\$ 25,972$ & $\$ 9,254$ & $64 \%$ \\
\hline & II & $\$ 70,104$ & $\$ 41,373$ & $41 \%$ \\
\hline \multirow{4}{*}{ PET scan } & All & $\$ 55,421$ & $\$ 43,035$ & $22 \%$ \\
\hline & 0 & $\$ 3,577$ & $\$ 5,429$ & $-52 \%$ \\
\hline & I & $\$ 32,459$ & $\$ 16,929$ & $48 \%$ \\
\hline & II & $\$ 135,076$ & $\$ 110,164$ & $18 \%$ \\
\hline
\end{tabular}




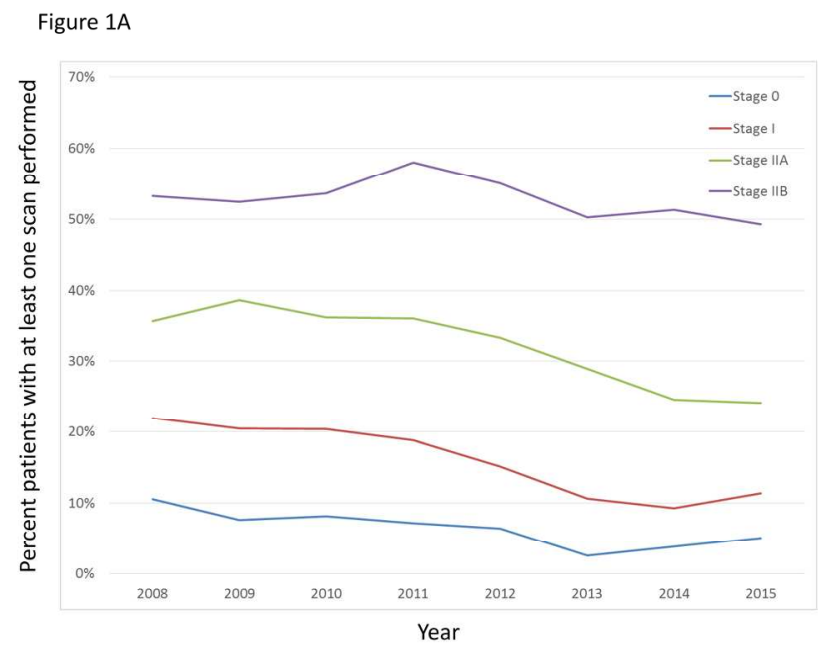

Percentage of patients who had at least one scan performed, by year. Each line represents a different disease stage (blue: stage 0, red: stage I, green: stage IIA, purple: stage IIB). A) At least one scan of any imaging modality.

Figure $1 \mathrm{~A}$

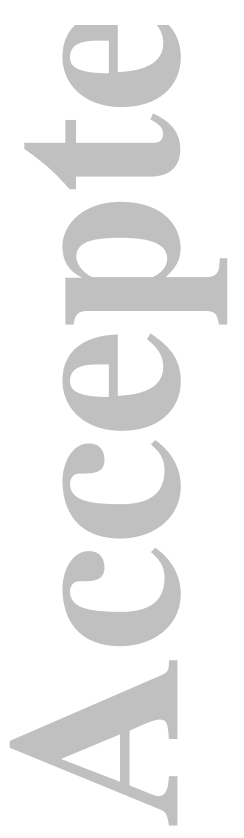

$190 \times 107 \mathrm{~mm}(300 \times 300 \mathrm{DPI})$ 
Figure 1B

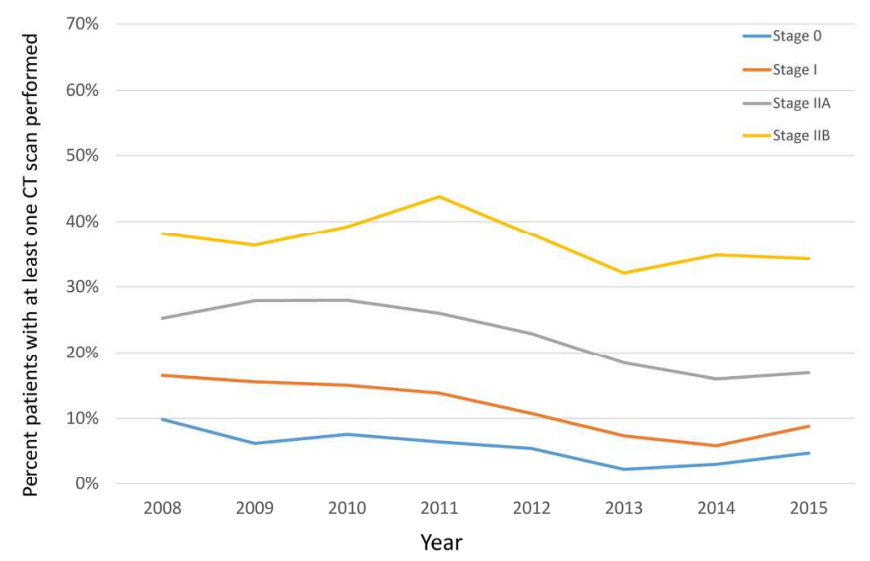

Percentage of patients who had at least one scan performed, by year. Each line represents a different disease stage (blue: stage 0, red: stage I, green: stage IIA, purple: stage IIB). B) At least one CT scan. Figure $1 \mathrm{~B}$

$190 \times 107 \mathrm{~mm}(300 \times 300 \mathrm{DPI})$

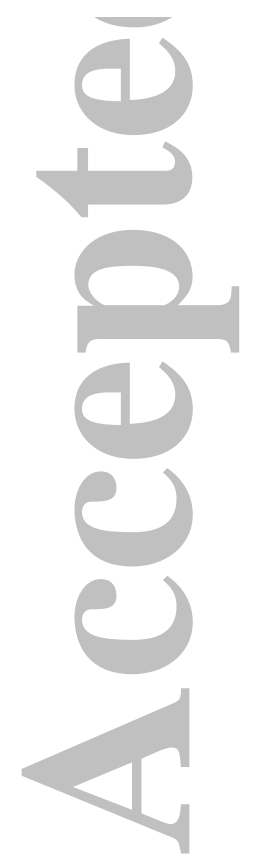




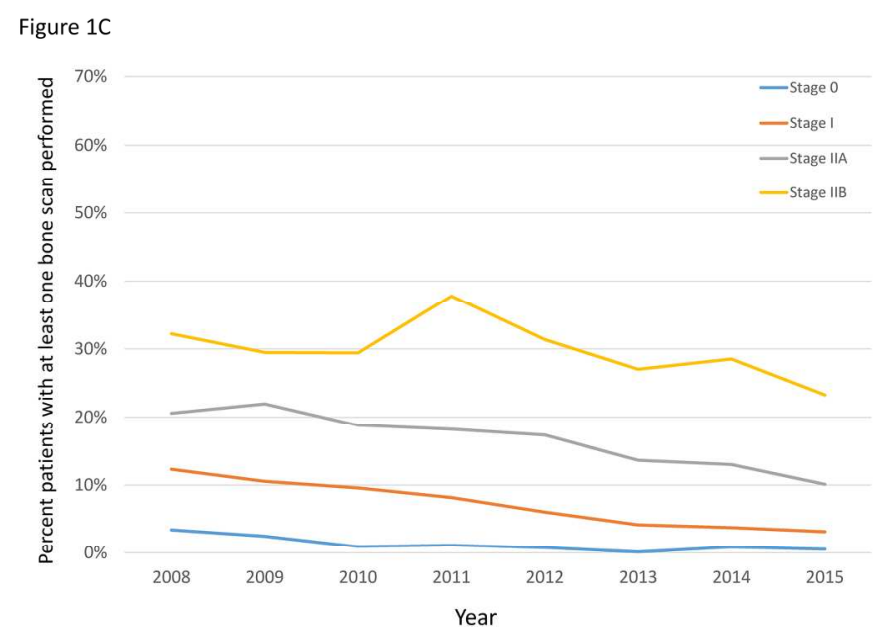

Percentage of patients who had at least one scan performed, by year. Each line represents a different disease stage (blue: stage 0, red: stage I, green: stage IIA, purple: stage IIB). C) At least one bone scan. Figure $1 \mathrm{C}$

$190 \times 107 \mathrm{~mm}(300 \times 300 \mathrm{DPI})$

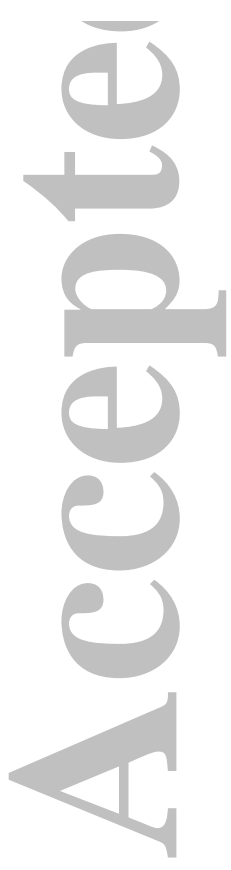




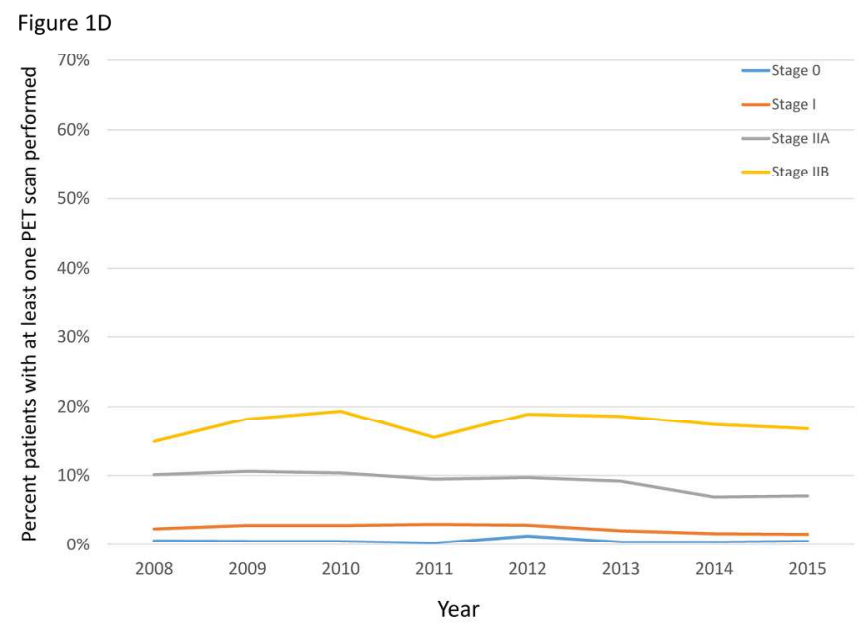

Percentage of patients who had at least one scan performed, by year. Each line represents a different disease stage (blue: stage 0, red: stage I, green: stage IIA, purple: stage IIB). D) At least one PET scan. Figure 1D

$190 \times 107 \mathrm{~mm}(300 \times 300 \mathrm{DPI})$

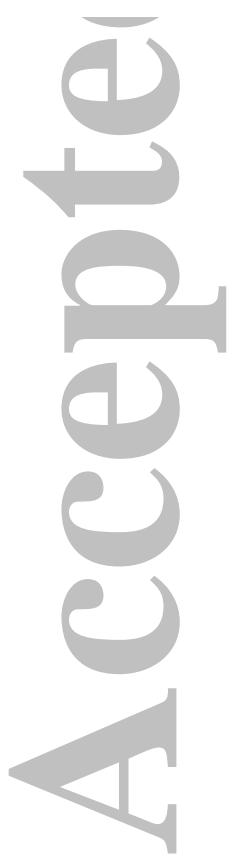


Figure 2

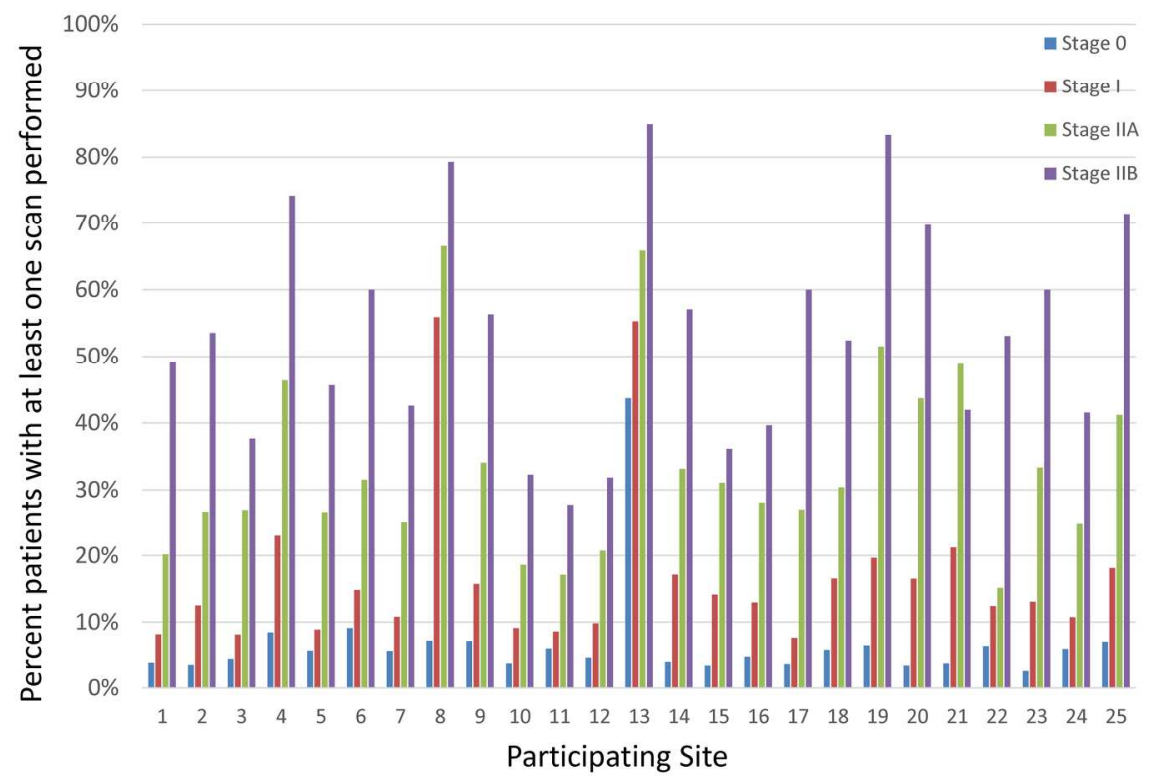

Percentage of patients who had at least one scan performed, by participating site. Sites are listed on the $\mathrm{x}$ axis from 1-25. Bars represent different disease stages (blue: stage 0, red: stage I, green: stage IIA, purple: stage IIB).

Figure 2

$190 \times 142 \mathrm{~mm}(300 \times 300 \mathrm{DPI})$ 
Supplemental Figure 1.

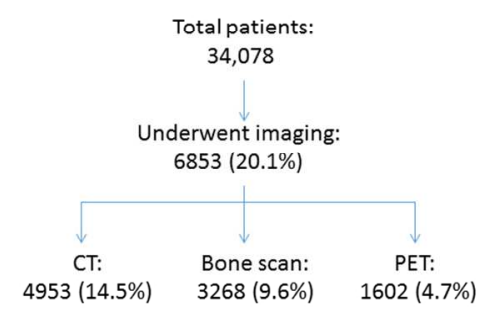

Diagram of number of patients diagnosed between 2008-2015 who underwent at least one imaging test. Supplemental Figure 1

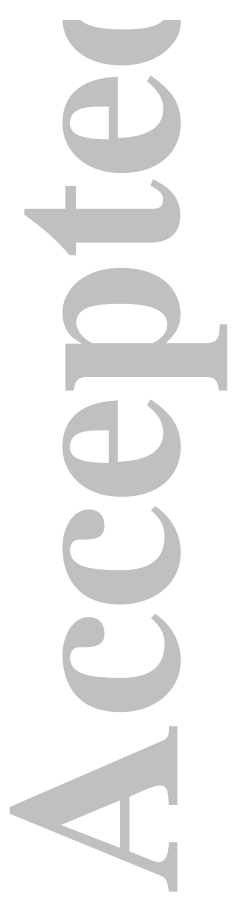
$338 \times 190 \mathrm{~mm}(96 \times 96 \mathrm{DPI})$ 
Supplemental Figure 2

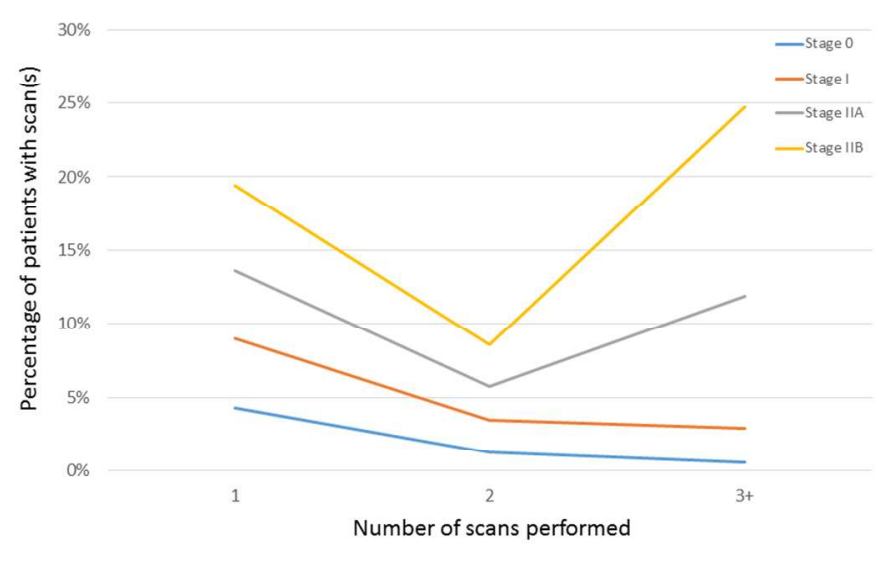

Percentage of patients who had scans performed, by stage. On the $\mathrm{x}$ axis it denotes patients who had 1,2 , or 3 or more scans performed. Each line represents a different disease stage (blue: stage 0, red: stage I, green: stage IIA, purple: stage IIB).

Supplemental Figure 2

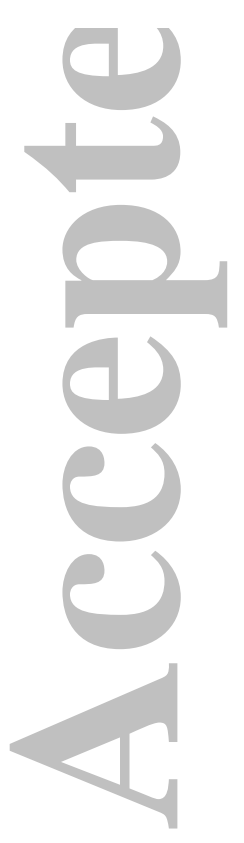

$338 \times 190 \mathrm{~mm}(96 \times 96$ DPI) 
Supplemental Figure 3A. Stage 0

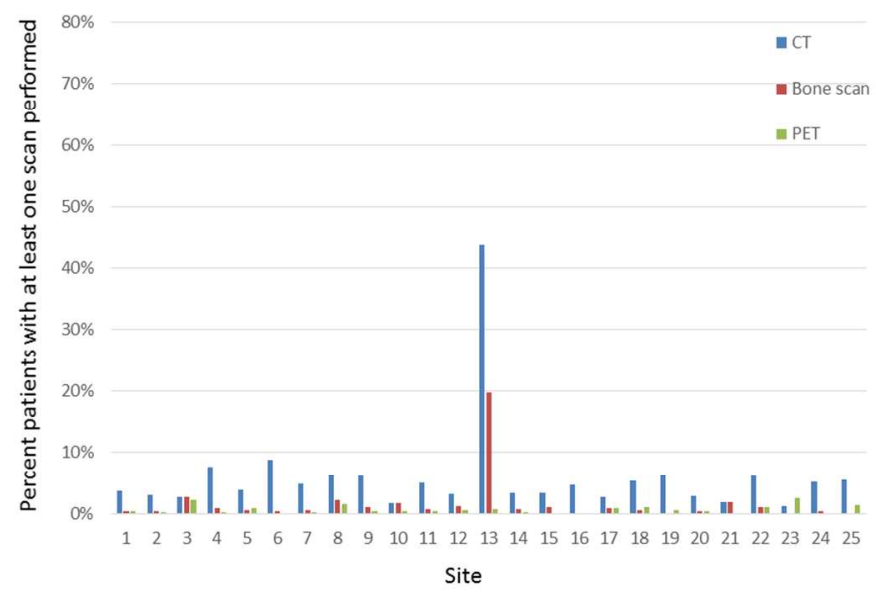

Percentage of patients who had at least one scan performed, by participating site. Sites are listed on the $x$ axis from 1-25. Bars represent different imaging modalities (blue: CT, red: bone scan, green: PET). A) Stage 0.

Supplemental Figure $3 \mathrm{~A}$

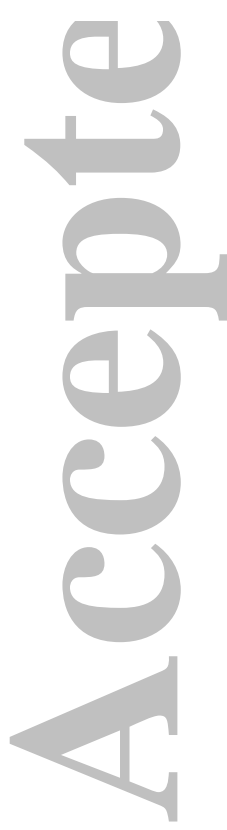
$338 \times 190 \mathrm{~mm}(96 \times 96 \mathrm{DPI})$ 


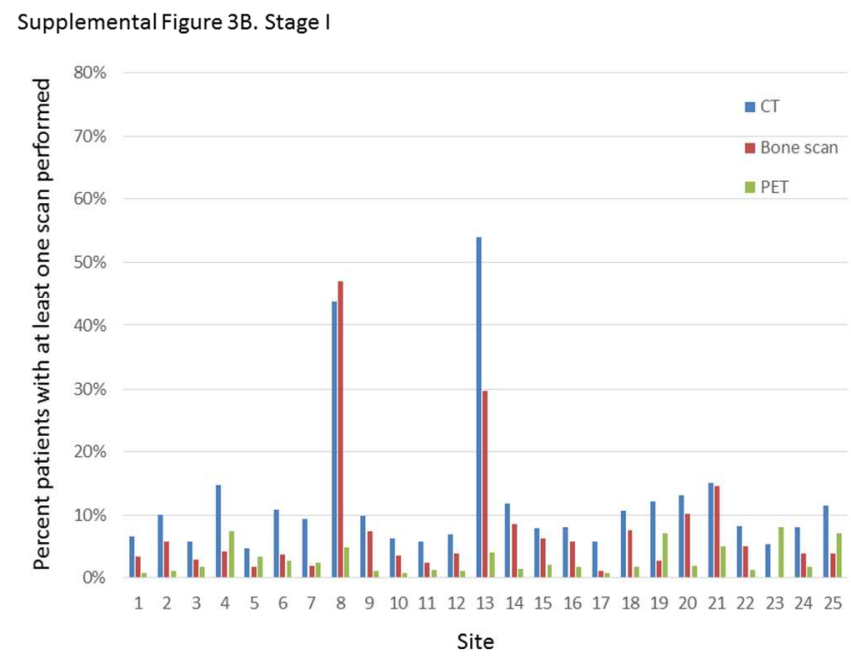

Percentage of patients who had at least one scan performed, by participating site. Sites are listed on the $\mathrm{X}$ axis from 1-25. Bars represent different imaging modalities (blue: CT, red: bone scan, green: PET). B) Stage I.

Supplemental Figure 3B

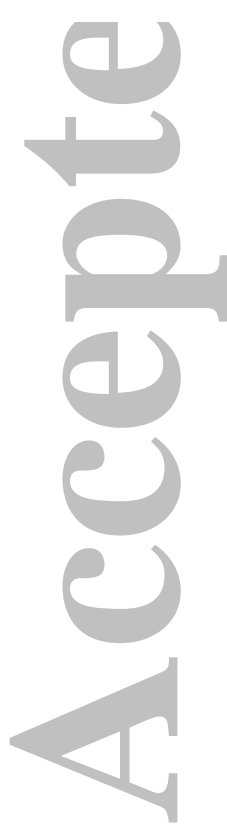
$338 \times 190 \mathrm{~mm}$ (96 x 96 DPI) 


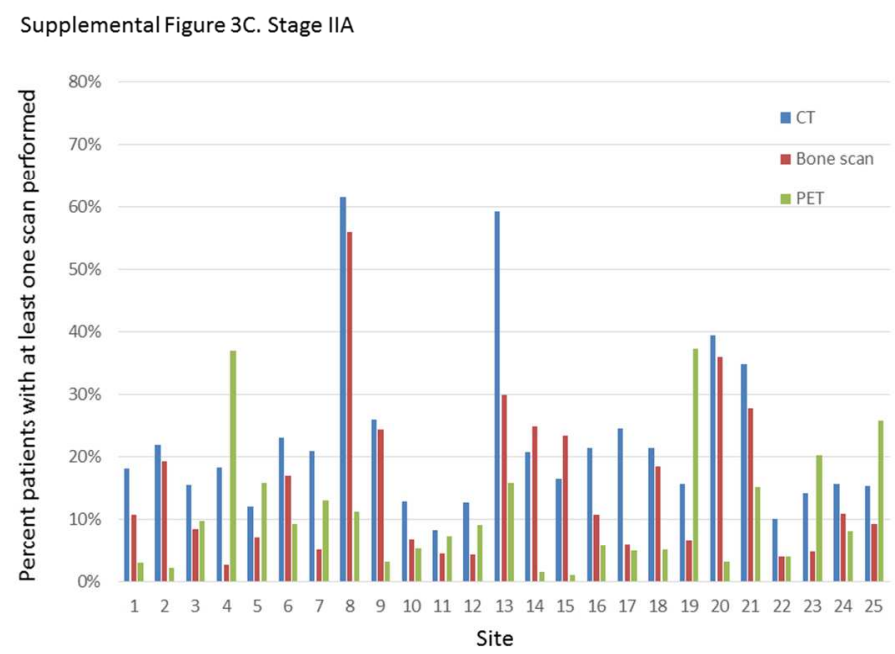

Percentage of patients who had at least one scan performed, by participating site. Sites are listed on the $\mathrm{x}$ axis from 1-25. Bars represent different imaging modalities (blue: CT, red: bone scan, green: PET). C) Stage IIA.

Supplemental Figure 3C

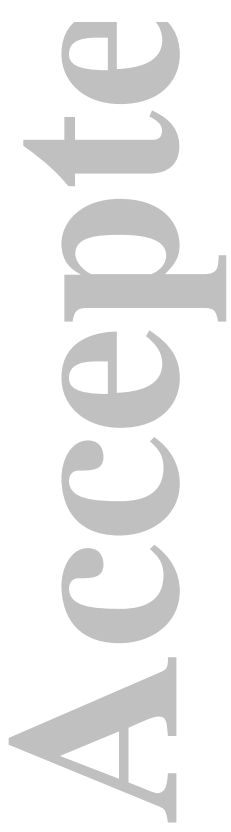
$338 \times 190 \mathrm{~mm}(96 \times 96 \mathrm{DPI})$ 
Supplemental Figure 3D. Stage IIB

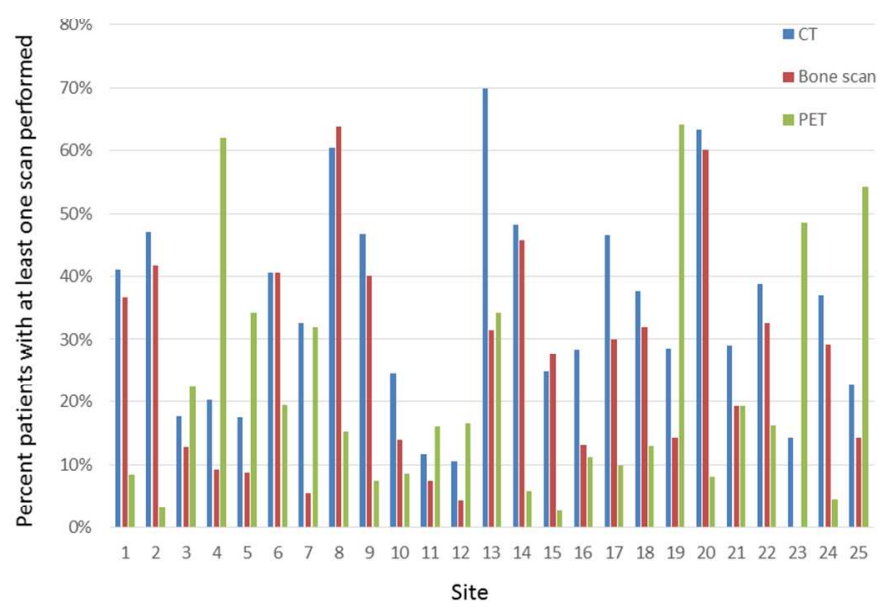

Percentage of patients who had at least one scan performed, by participating site. Sites are listed on the $x$ axis from 1-25. Bars represent different imaging modalities (blue: CT, red: bone scan, green: PET). D) Stage IIB.

Supplemental Figure 3D

$338 \times 190 \mathrm{~mm}$ (96 x 96 DPI)

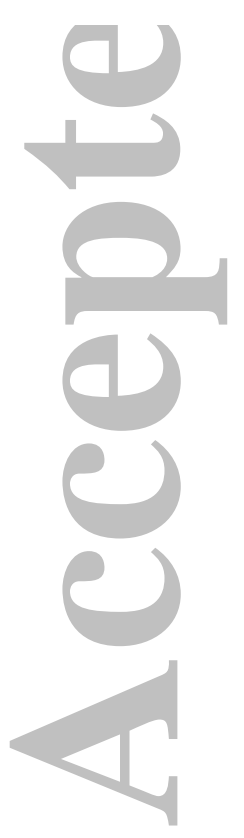


Supplemental Table 1. Change in percentage of patients who underwent at least one scan in 2008 and 2015, by stage and type of imaging test.

\begin{tabular}{|c|c|c|c|c|}
\hline Stage & Type of scan & 2008 & 2015 & P value \\
\hline \multirow[t]{4}{*}{0} & At least one scan & $11 \%$ & $5 \%$ & $<0.001$ \\
\hline & CT scan & $10 \%$ & $5 \%$ & $<0.001$ \\
\hline & Bone scan & $3 \%$ & $1 \%$ & $<0.001$ \\
\hline & PET scan & $0.57 \%$ & $0.51 \%$ & 0.89 \\
\hline \multirow[t]{4}{*}{$\mathbf{I}$} & At least one scan & $22 \%$ & $11 \%$ & $<0.001$ \\
\hline & CT scan & $16 \%$ & $9 \%$ & $<0.001$ \\
\hline & Bone scan & $12 \%$ & $3 \%$ & $<0.001$ \\
\hline & PET scan & $2.3 \%$ & $1.5 \%$ & $<0.001$ \\
\hline \multirow[t]{4}{*}{ IIA } & At least one scan & $36 \%$ & $24 \%$ & $<0.001$ \\
\hline & CT scan & $25 \%$ & $17 \%$ & $<0.001$ \\
\hline & Bone scan & $21 \%$ & $10 \%$ & $<0.001$ \\
\hline & PET scan & $10 \%$ & $7 \%$ & $<0.001$ \\
\hline \multirow[t]{4}{*}{ IIB } & At least one scan & $53 \%$ & $49 \%$ & 0.10 \\
\hline & CT scan & $38 \%$ & $34 \%$ & 0.75 \\
\hline & Bone scan & $32 \%$ & $23 \%$ & 0.002 \\
\hline & PET scan & $15 \%$ & $17 \%$ & 0.75 \\
\hline
\end{tabular}

